\title{
Progress in Antimonide Based III-V Compound Semiconductors and Devices
}

\author{
Chao Liu, Yanbo Li, Yiping Zeng \\ Key Laboratory of Semiconductor Materials Science, Institute of Semiconductors, Chinese Academy of Sciences, \\ Beijing, China \\ E-mail: cliu@semi.ac.cn \\ Received December 2, 2009; revised February 11, 2010; accepted February 15, 2010
}

\begin{abstract}
In recent years, the narrow bandgap antimonide based compound semiconductors (ABCS) are widely regarded as the first candidate materials for fabrication of the third generation infrared photon detectors and integrated circuits with ultra-high speed and ultra-low power consumption. Due to their unique bandgap structure and physical properties, it makes a vast space to develop various novel devices, and becomes a hot research area in many developed countries such as USA, Japan, Germany and Israel etc. Research progress in the preparation and application of ABCS materials, existing problems and some latest results are briefly introduced.
\end{abstract}

Keywords: Antimonide Based Compound Semiconductors (ABCS), IR Laser, IR Detector, Integrated Circuit, Functional Device

\section{Introduction}

Antimonide based compound semiconductors (ABCS) mainly refer to the antimonide based binary, ternary and quaternary compound semiconductor materials, consisting of the III-group elements (Ga, In, Al, etc.) and $\mathrm{Sb}, \mathrm{As}$ and other V-group elements, such as $\mathrm{GaSb}, \mathrm{InSb}, \mathrm{Al}-$ $\mathrm{GaSb}$, InAsSb, AlGaAsSb, InGaAsSb and so on. Their crystal lattices are around $6.1 \AA$ and they together with the InAs-based materials have been routinely called the "6.1 ̊ III-V family materials". Antimonide based semiconductors with narrow bandgap as the basic feature, in the condition of lattice matched or nearly matched with strain with $\mathrm{GaSb}$, InAs, InP and other commonly used substrates, their bandgap can be adjusted in a wide range coveraging from near-infrared wavelength $0.78 \mu \mathrm{m}$ (AlSb) to far-infrared spectral regions $12 \mu \mathrm{m}$ (InAsSb). The heterojunctions formed between them can have type-I, type-II staggered and type-II misaligned band lineups. The unique band structure and excellent physical properties of ABCS based materials provide great freedom and flexibility for band engineering and structural design of materials and create a broad space for development of high-performance microelectronics, opto-electronic devices and integrated circuits. Applications could include active-array space-based radar, satellite communications, ultra-high-speed and ultra-low power integrated circuits, portable mobile devices, gas environmental monitoring, chemical detection, bio-medical diagnosis, drug analysis and other fields [1-8].

\section{The Physical Properties and Preparation Technology of ABCS Based Materials}

The in-depth study of antimonide based semiconductor materials and devices applications was rapidly developed in recent ten years. Especially after the antimonide based compound semiconductors program (ABCS program) [9] was launched by Defense Advanced Research Projects Agency (DARPA) of USA in 2001, a series of important developments and breakthroughs have been made in the study of antimonide based microstructure materials and device applications worldwide. The narrow bandgap antimonide based compound semiconductors are widely regarded as the first candidate materials for fabrication of the third generation infrared photon detectors and integrated circuits with ultra-high speed and ultra-low power consumption and also as the important materials for middle and far infrared quantum cascade lasers and thermophotovoltaic cells suitable for medium and low temperature heat sources.

The comparison of physical properties of III-V compound semiconductors (at RT) is showed in Table 1. We 
Table 1. Comparison of physical properties of III-V compound semiconductors (at RT).

\begin{tabular}{|c|c|c|c|c|c|c|c|}
\hline Physical properties & InSb & GaSb & AlSb & InAs & GaAs & InP & GaN \\
\hline Energy gap/(eV) & 0.18 & 0.70 & 1.63 & 0.36 & 1.42 & 1.35 & 3.39 \\
\hline $\begin{array}{l}\text { Electron mobility } / \\
\left(\mathrm{cm}^{2} / \mathrm{V} . \mathrm{s}\right)\end{array}$ & $8 \times 10^{4}$ & 5000 & 200 & $3 \times 10^{4}$ & 8500 & 5400 & 900 \\
\hline $\begin{array}{l}\text { Electron saturation velocity } \\
\qquad\left(\times 10^{7} \mathrm{~cm} / \mathrm{s}\right)\end{array}$ & 4.0 & - & - & 4.0 & 1.0 & 1.0 & 2.7 \\
\hline $\begin{array}{c}\text { Electron mean free path } \\
\text { length/nm }\end{array}$ & 226 & - & - & 194 & 80 & - & - \\
\hline $\begin{array}{cc}\text { Effective mass } & \text { Electron } \\
\left(\mathrm{m}_{0}\right) & \text { Hole }\end{array}$ & $\begin{array}{c}0.014 \\
0.018(\mathrm{~L}) \\
0.4(\mathrm{H})\end{array}$ & $\begin{array}{c}0.042 \\
0.4\end{array}$ & $\begin{array}{l}0.12 \\
0.98\end{array}$ & $\begin{array}{c}0.024 \\
0.025(\mathrm{~L}) \\
0.37(\mathrm{H})\end{array}$ & $\begin{array}{c}0.067 \\
0.082(\mathrm{~L}) \\
0.45(\mathrm{H})\end{array}$ & $\begin{array}{c}0.077 \\
0.12(\mathrm{~L}) \\
0.55(\mathrm{H})\end{array}$ & $\begin{array}{l}0.2 \\
0.6\end{array}$ \\
\hline $\begin{array}{l}\text { Thermal conductivity } \\
\text { (W/cm.K) }\end{array}$ & 0.15 & 0.4 & 0.7 & 0.27 & 0.5 & 0.7 & 1.3 \\
\hline Relative dielectric constant & 17.9 & 15.7 & 12.04 & 15.1 & 12.8 & 12.5 & 9 \\
\hline
\end{tabular}

can see that ABCS have excellent physical properties. For example the InSb has the smallest bandgap, the smallest effective mass of carriers, the largest electronic saturation drift velocity and mobility of any III-V compound semiconductor materials. The relationship between energy gap \& spectral wavelength and lattice constant is shown in Figure 1 which also shows the evolution of HEMTs and HBTs transistors for higher frequencies and lower power operation. The relative position between energy gap and band offset of III-V semiconductors is shown in Figure 2. Thus it can be seen that there is a considerable band offset and a rich structure of the energy band alignment in the ABCS heterojunctions. By regulating the compositions of ABCS multiple compounds, it is convenient to carry out the bandgap engineering of novel devices in the condition of the lattice match or the strained match.

Antimonide based compound semiconductors can generally be divided into bulk crystals and film materials. The most common bulk crystals are GaSb、InSb and InAs. Due to the relatively low melting point of $\mathrm{GaSb}$ and $\mathrm{InSb}$, i.e., $712{ }^{\circ} \mathrm{C}$ and $525{ }^{\circ} \mathrm{C}$ respectively, no dissociation near melting point temperature and small saturation vapor pressure, they can be prepared using the horizontal Bridgman growth of zone melting or vertical drawn VP method which is similar to the growth of Ge bulk crystal. While the InAs (melting point $943^{\circ} \mathrm{C}$ ) bulk crystal can be grown using liquid covering Czochralski (LEC) Pulling Method or vertical gradient freeze (VGF) method which is similar to the growth of GaAs bulk crystal. Because of their small bandgap, at room temperature, ABCS's intrinsic carrier concentration are too high to get high resistivity (semi-insulating) substrate materials which is a serious impediment to the ABCS's applications in the field of microelectronic devices. At present the ultra-high pure InSb bulk crystal's carrier concentration can be less than $10^{13} / \mathrm{cm}^{3}$ and the residual hole concentration of GaSb bulk crystals is about $2 \times$
$10^{16} / \mathrm{cm}^{3}$. Because the growth process is very immature and there is immiscible gap in the multi-elements antimonide, the ternary, quaternary antimonide bulk crystal materials are rarely used.

The commonly used methods for preparation of antimonide film materials are liquid phase epitaxy (LPE), molecular beam epitaxy (MBE) and metal organic chemical vapor deposition (MOCVD or OMVPE). LPE method has the advantages of relatively simple process, less expensive epitaxial equipment, high utilization rate of the source material, high crystalline quality of the epitaxial films, fast growing, particularly suitable for the preparation of thick-film materials and so on. LPE method is a near-thermodynamic equilibrium growth technology, and therefore can not be used for the growth of the metastable ternary, quaternary antimonide materials whose components in the immiscible gap. Its growth rate is generally higher than MOCVD and MBE, and changes from different substrate crystalline phases with the typical growth rate of $100 \mathrm{~nm} / \mathrm{min}$ to a few $\mu \mathrm{m} / \mathrm{min}$. The weakness of LEP is that it can not be used for precision controlled growth of very thin films of nano-scale. That is to say that it is not applicable to the growth of superlattices or quantum-well devices and other complex micro-structure materials. In addition, the morphology of materials grown by LPE is usually worse than that grown by MOCVD or MBE. In recent years, a new method which combines LPE with $\mathrm{Zn}$ diffusion technology for low-cost, high efficient GaSb based InGaAsSb homogeneous pn junction thermophotovoltaic (TPV) cells has been developed [8]. This method first grows lattice matched $\mathrm{n}-\mathrm{In}_{0.15} \mathrm{Ga}_{0.85} \mathrm{As}_{0.17} \mathrm{Sb}_{0.83}(0.55 \mathrm{eV})$ epitaxial layer on the Te-doped n-type GaSb substrate associated with the LPE, then forms the pn homojunction in the InGaAsSb layer using $\mathrm{Zn}$ diffusion method. The external quantum efficiency of the TPV is as high as $90 \%$ at $2 \mu \mathrm{m}$ radiation wavelength and the cut-off wavelength is 2.3 $\mu \mathrm{m}$, very close to the technical parameters of materi- 


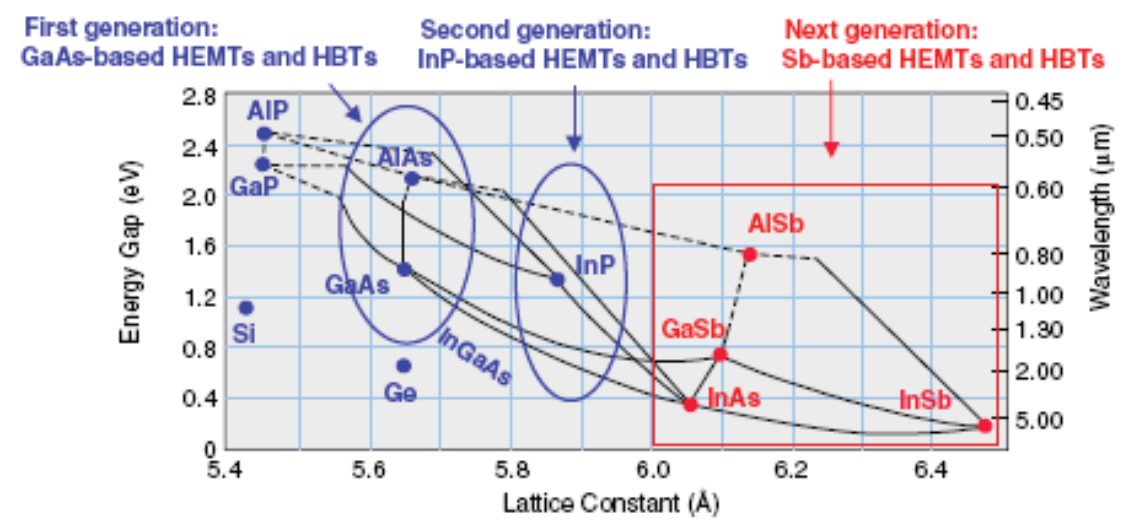

Figure 1. Energy gap \& spectral wavelength versus lattice constant, showing the evolution of HEMTs and HBTs transistors for high-frequency and low-power operation [1].

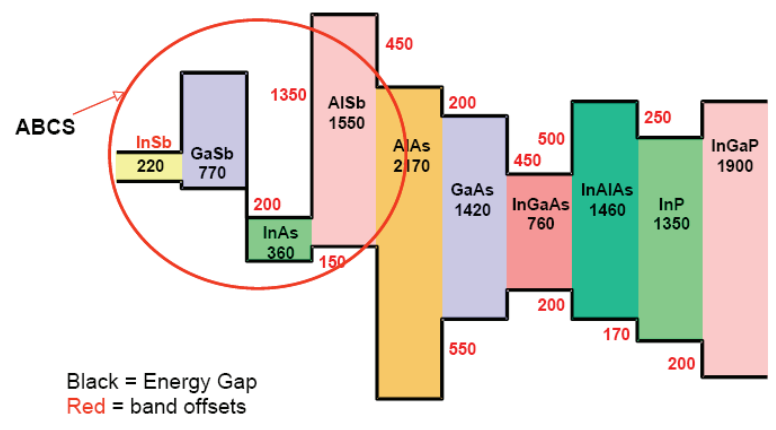

Figure 2. Relative position between energy gap and band offset of III-V semiconductors.

als grown by MOCVD or MBE. In addition, LPE method is also used to grow materials of mid-infrared InGaAsSb, InSb-based infrared detectors, LED and LD. It is a relatively mature, high efficiency, low cost growth technology which is easy to realize the industrialization.

Both MOCVD and MBE are low temperature epitaxial growth technology of non-thermodynamic equilibrium. You can grow almost all compositions of the multielements compound thin films including the ternary, quaternary antimonide which is in the immiscible-gap and in the metastable state. Both of them can be used for growth of complex micro-structural materials of ultrathin layers and is very suitable for development of new optoelectronic devices and circuits. Antimonide based materials grown by either MOCVD or MBE have their own characteristics. For a specific device structure, it is still hard to judge which growth method used for growth of the device structure is better. In general, MOCVD is suitable for mass production of epitaxial materials whose device structure is relatively mature and easy to expand the size and production capacity. While the MBE is more suitable for research and development of the novel epitaxial materials with hyperfine and complex structures. Although production-based MBE equipment has been developed, it is still not economical using the MBE for mass production when considering the cost.
The first epitaxial growth of antimonides thin film materials using MOCVD was done by Manasevit and Simpson in 1969 who used $\mathrm{TMGa}$ and $\mathrm{SbH}_{3}$ (stibine) source for growing GaSb films [4]. Different from epitaxial materials grown by MBE, The types of metallorganics have a critical influence on the quality of epitaxial materials grown by MOCVD. The commonly used III-group metal-organic sources by MOCVD for antimonide based compounds are 3-methyl compound and 3-ethyl compound, such as: TMGa, TMIn, TMAl, TEGa, TEIn, etc. The commonly used V-group sources are $\mathrm{TMSb}, \mathrm{AsH}_{3}, \mathrm{PH}_{3}, \mathrm{TMBi}$ and $\mathrm{RF}-\mathrm{N}_{2}$, etc. Antimonides are generally low melting point materials and the temperature of epitaxial substrate is generally about $500^{\circ} \mathrm{C}$. In addition to TMIn's lower decomposition temperature $\left(250-300^{\circ} \mathrm{C}\right)$, the majority of III-group metal-organic sources can not be completely decomposed below $500^{\circ} \mathrm{C}$. Therefore, to growing InSb material whose melting point is only $525^{\circ} \mathrm{C}$, new organic source material with a lower decomposition temperature must be adopted. At present the new organic sources which have been successfully applied for growing antimonides by MOCVD are: TDMASb (trisdimethylaminoantimony, decomposition temperature $<300^{\circ} \mathrm{C}$ ), TBDMSb, TASb (triallyantimony), TMAA (trimethylamine alane), TTBAl (tritertiarybutylaluminum), EDMAA ( ethyldimethylaminealane) and so on. In addition, because of the lack of room temperature chemical stabilized antimony hydride $\left(\mathrm{SbH}_{3}\right)$, when growing Al-containing antimonide materials (such as: AlSb, AlGaSb, AlGaAsSb, etc.), it is easy to appear carbon and oxygen contamination problem. This phenomenon may be related to the lack of active hydrogen atoms on the surface of epitaxial materials in which $\mathrm{C}$ is general for p-type doping. Even if the Al content in the alloy is only $20 \%$, the doping concentrations of $\mathrm{C}$ and $\mathrm{O}$ can reach more than $1 \times 10^{18} / \mathrm{cm}^{3}$ in the epitaxial film. This causes certain difficulties in growing n-type doping Al-containing antimonide films. The presence of high concentration of $\mathrm{O}$ impurity in Al-containing antimonide materials will make these materials have the semi- 
insulating properties and difficult to measure their electrical properties. The origin of $\mathrm{O}$ impurity is very complex, and both the purity of the metal organic sources and the epitaxial environment and process conditions are closely related. The development of new organic aluminum source such as TMAA, TTBAl, EDMAA etc. is precisely in order to inhibit the serious $C$ contamination problem [4-5]. Thus, growing AlSb and their multielement materials using MOCVD is the most challenging work in all the III-V epitaxial materials technologies.

The epitaxial growth of antimonide materials using MBE was following earlier pioneering work of the IBM group of L.L Chang and L. Esak, first on InAs/GaSb and InAs/AlSb films [3]. Different from MOCVD process, MBE uses ultra-high vacuum epitaxial environment with single-element materials for molecular beam sources and is easy to implement epitaxy of atomic layer and in situ real-time monitoring, avoiding the $\mathrm{C}$-pollution problem which exits in Al-containing materials growing by MOCVD and greatly reducing the concentration of $\mathrm{O}$ doping. In fact most of the prototype devices having complex fine structures and low-dimensional structures (quantum wells, quantum wires and quantum dots) were first achieved using materials grown by MBE. It is noteworthy that, no matter MOCVD or MBE method, the use of substrates whose surface orientation have a small angle offset (i.e., low-density atomic step on the surface of the substrate) seem to be more accessible high-quality epitaxial layers. Experiments confirmed that the use of $\mathrm{GaSb}(100)$ substrates miscut $2^{\circ}$ towards (110) or $6^{\circ}$ towards (1i1) B may get higher crystal quality of InGaAsSb and AlGaAsSb epitaxial layers [5]. To overcome the difficulty that antimonides have no semi-insulating substrate materials, the use of GaAs, Si and other heterogeneous substrate materials for epitaxy of ABCS films have also attracted great attention. H. Toyota, etc. [10] reported that they grown high-quality GaSb/AlGaSb multi-quantum well (MQW) structures with a 5nm AlSb initiation layer and a relatively thick $\mathrm{GaSb}$ buffer layer $(0.5-2.0 \mu \mathrm{m})$ grown on $\mathrm{Si}(001)$ substrates by molecular beam epitaxy. The photoluminescence (PL) emission around $1.55 \mu \mathrm{m}$ wavelength was observed for $\mathrm{GaSb} /$ $\mathrm{AlGaSb} \mathrm{MQW}$ structure at room temperature. Low dislocation density, high-quality GaSb epitaxial films on GaAs (001) substrates stripe-patterned with $\mathrm{SiO}_{2}$ is also prepared by MOCVD with low temperature epitaxial lateral overgrowth (ELO) method [11].

Apart from common binary, ternary and quaternary antimonides being composed of $\mathrm{Al}, \mathrm{Ga}$, In, $\mathrm{As}$ and $\mathrm{Sb}$, in order to extend the applications of antimonide-based materials in the far-infrared band $(>5 \mu \mathrm{m})$, easy to adjust the material lattice constant to match the substrates' lattice constant of GaSb, InAs et al. and develop new functional materials, recently some ternary, quaternary antimonides containing $\mathrm{N}(<2 \%), \mathrm{P}$ or $\mathrm{Bi}(<2 \%)$ and fiveelements antimonides such as AlGaInAsSb, GaInNAsSb etc have also aroused people's concern and research interest [12-14]. T. Ashlet, etc. [12] found that the addition of a small percentage of nitrogen $(<2 \%)$ to $\mathrm{GaSb}, \mathrm{InSb}$, and $\mathrm{GaInSb}$ materials would significantly change their energy band structures (bandgap become smaller) which is very conducive to develop multi-band infrared detectors.

\section{Application of ABCS Materials}

The early focus of antimonide based compound semiconductors comes from its application prospect in midand far-infrared (photon) detectors, but the first to enter the market and get a large-scale industrial production is high-sensitivity InSb magnetoresistive Hall sensors. In 2004, Asahi Kasei Electronic (AKE) of Japan which account for $70 \%$ of the global market share of Hall sensors announced that its InSb Hall sensor output had reached more than 100 million per month. These products are widely used in small brushless DC motors, automotive electronics and consumer electronics products and other fields. InSb-based infrared detector arrays have gained a market dominant position of ground-infrared applications and space instrumentation fields. In addition to these more mature products, antimonide materials have made great progress in the third-generation infrared focal plane array detectors, mid and far infrared quantum cascade lasers, quantum dot lasers, ultra-high-speed, ultra-lowpower and low-noise amplifiers, thermophotovoltaic devices and so on in recent years. The following describes some latest results and trends of development of application of ABSC materials.

\subsection{Microelectronic Devices and Integrated Circuits}

HEMT and HBT devices and circuits used by millimeter-wave radar and high-frequency digital communications have so far experienced first generation based on GaAs-based materials, second generation based on InP-based materials and is currently to the development of third generation of HEMT and HBT devices and circuits based on antimonide based compound materials with ultra-high speed, ultra-lower power consumption and noise factor. After DARPA launched the ABCS projects in 2001, Rockwell Scientific Company (RSC) starting in 2003, has developed Ka-band (34-36 GHz), W-band (92-102 GHz) and X-band (8-12 GHz) low noise amplifier microwave monolithic integrated circuit (MMIC) and the transmit/receive (T/R) integrated modules based on InAs/AlSb mHEMT through its mature GaAs pHEMT technology platform. Currently ABCS Integrated Circuit was regarded as a core and key technologies to accelerate the development by DARPA and the short-term goal is to develop practical ABCS IC 
products with integration of transistors more than 5000 and the working voltage of about $0.5 \mathrm{~V}$.

The five-stage W-band MMIC LNA chip is shown in Figure 3 [15]. The compact $1.2 \mathrm{~mm}^{2}$ five-stage W-band LNA using $0.2-\mu \mathrm{m}$ gate length InAs/AlSb metamorphic HEMTs demonstrated a $3.9 \mathrm{~dB}$ noise-figure at $94 \mathrm{GHz}$ with an associated gain of $20.5 \mathrm{~dB}, \mathrm{f}_{\mathrm{T}}=142 \mathrm{GHz}, \mathrm{f}_{\max }=$ $178 \mathrm{GHz}$. The measured dc power dissipation of the ABCS LNA was only $6.0 \mathrm{~mW}$ which is less than onetenth the dc power dissipation of a typical equivalent InGaAs/AlGaAs/GaAs HEMT LNA. The ABCS HEMT structure [15] is grown using MBE on semi-insulating GaAs substrates using an AlSb buffer to accommodate the lattice mismatch and a strained InAlAs cap layer to provide a chemically stable surface layer and minimize gate leakage. Hall measurements show 2DEG of InAs channel concentration and mobility to be $3.7 \times 10^{12} \mathrm{~cm}^{-2}$ and $19,000 \mathrm{~cm}^{2} / \mathrm{Vs}$ at $295 \mathrm{~K}$.

Growing the Sb-based HEMTs on Si substrate can combine the high mobility of antimonide based compound materials and excellent features of $\mathrm{Si}$ substrate with broad application prospects. M.K. Kwang et al. [16] reported their research results of growing AlGaSb/InAs HEMT structure on Si substrates. By using an $\mathrm{AlGaSb}$ buffer layer containing InSb quantum dots for dislocation termination, they can effectively terminate the propagation of micro-twin-induced structural defects into overlying layers, resulting in the low defect material grown on a largely mismatched substrate with a relatively thin buffer layer. Figure 4 shows the schematic of the Al$\mathrm{GaSb} / \mathrm{InAs}$ HEMT grown on Si substrate. The high quality $\mathrm{AlGaSb} / \mathrm{InAs} \mathrm{HEMT}$ materials grown on $\mathrm{Si}$ (001) substrate with the electron mobility of higher than 16000 $\mathrm{cm}^{2} \mathrm{~V}^{-1} \mathrm{~s}^{-1}$ at room-temperature and a sheet density of 2.5 $\times 10^{12} \mathrm{~cm}^{-2}$ were obtained by using this technique. It seems to provide a new way of integrating Sb-based devices and circuits on $\mathrm{Si}$ substrate.

\subsection{Infrared Detectors}

There has been more than 60 years in the study of the infrared photon detectors. The development of the first

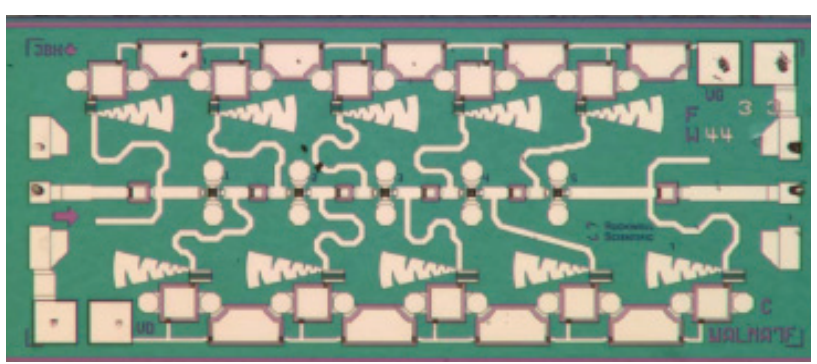

Figure 3 A photomicrograph of the five-stage ABCS HEMT MMIC W-band LNA fabricated by Rockwell Scientific Company.

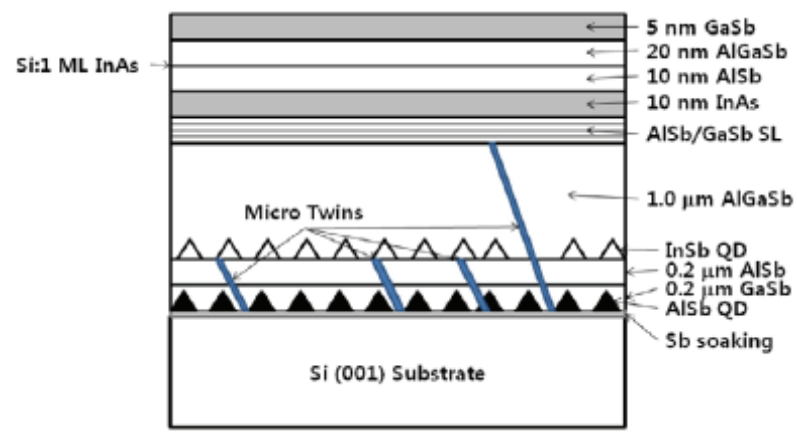

Figure 4. Schematic of the AIGaSb/InAs HEMT grown on Si substrate [16].

generation of infrared detectors began in the late forties of the last century, using one-dimensional linear arrays which were made of lead salt such as PbSe, and PbTe to detect the mid-infrared (MWIR) $(3-5 \mu \mathrm{m})$. The second generation infrared detector materials were mainly $\mathrm{InSb}$ and $\mathrm{HgCdTe}(\mathrm{MCT})$ for the two atmospheric IR windows of the mid-infrared band and the far-infrared band (LWIR) respectively [17]. The devices with the focal plane array structures of one dimension and two dimensions are currently very widely used and more mature products. In recent years, the third generation infrared detectors were researched and developed in many countries, their main features are multi-band infrared detection, high-resolution (high pixels and high frame rate), high operating temperatures, high spatial uniformity, high stability and low cost [18]. As it is difficult for the MCT to achieve large area uniformity and stability, the ABCS superlattice materials is generally considered as the preferred materials of the third-generation infrared detectors [6-7]. In principle, the bandgap of the ABCS superlattice materials can be tailored to cover the entire spectrum area of infrared detection by adjusting the thickness and composition of the ABCS materials [19].

In 2007, C.J. Hill et al. of the Jet Propulsion Laboratory [20] reported the GaSb/InAs type-II superlattice detectors grown on unintentional doped p-type $\mathrm{GaSb}$ (100) substrate designed for $2-5 \mu \mathrm{m}$ and $8-12 \mu \mathrm{m}$ bands infrared absorption. The LWIR detectors have detectivities as high as $8 \times 10^{10}$ Jones $\left(\mathrm{cm} . \mathrm{H}^{1 / 2} / \mathrm{W}\right)$ with a differential resistance-area product (RoA) greater than $6 \mathrm{Ohm}$ $\mathrm{cm}^{2}$ at $80 \mathrm{~K}$ with a cutoff wavelength of approximately $12 \mu \mathrm{m}$. The measured internal quantum efficiency $\left(\mathrm{QE}_{\mathrm{i}}\right)$ of these front-side illuminated devices is close to $30 \%$ in the $10-11 \mu \mathrm{m}$ range. The MWIR detectors have detectivities as high as $8 \times 10^{13}$ Jones with a differential resistance-area product greater than $3 \times 10^{7} \mathrm{Ohm} \mathrm{cm}^{2}$ at $80 \mathrm{~K}$ with a cutoff wavelength of approximately $3.7 \mu \mathrm{m}$. The measured internal quantum efficiency of these front-side illuminated MWIR devices is close to $40 \%$ in the $2-3 \mu \mathrm{m}$ range at low temperature and increases to over $60 \%$ near room temperature. From the RoA and $\mathrm{QE}_{\mathrm{i}}$ indicators, we 
can see that the ABCS II-type superlattice mid-infrared detector will have a great potential for application of mid-infrared focal plane array devices of non-low-temperature environment. In addition, InAs/InGaSb type-II superlattice materials have also been widely concerned and in-depth research and they are considered as candidate materials for the third-generation infrared detectors.

Two-color or dual-band infrared detectors have the ability of inhibiting the complex background and improving the target detection efficiency and can significantly improve the system performances. Dual-band LWIR/VLWIR type-II superlattice infrared detectors was reported by E. H. Aifer et al. [21]. The cut-off wavelengths of the two bands are $11.4 \mu \mathrm{m}$ and $17 \mu \mathrm{m}$ respectively. But the quantum efficiency of the dual-band infrared detectors is too low (only 4-5\%) compared to the single-band type II superlattice infrared detectors and the device structure needs to be further optimized. High quality GaSb based two-color $288 \times 384$ MWIR InAs/ GaSb type-II SLS FPAs was reported by M. Münzberg et al. [22] of the Fraunhofer Institute in Freiburg. First, the "blue channel" consisting of 330 periods of p-type of a 7.5 ML InAs/10 ML GaSb was deposited on the GaSb substrate for spectral selective detection in the 3.0-4.1 $\mu \mathrm{m}$ wavelength range. Next, the "red channel" consisting of 150 periods of a $9.5 \mathrm{ML}$ InAs/10 ML GaSb superlattices was deposited for spectral selective detection in the 4.1-5.0 $\mu \mathrm{m}$ wavelength range. The thickness of the entire vertical pixel structure is only $4.5 \mu \mathrm{m}$, which significantly reduces the technological challenge compared to dual- band HgCdTe FPAs with a typical total layer thickness around $15 \mu \mathrm{m}$. Excellent thermal resolution with Noise Equivalent Temperature Difference (NETD) $<17 \mathrm{mK}$ for the "red channel" and NETD $<30 \mathrm{mK}$ for the "blue channel" has been achieved.

\subsection{Infrared Lasers}

Solid-infrared laser has important applications in gaseous environmental monitoring, chemical detection, bio-medical diagnosis, satellite remote sensing technology and so on. Antimonide based compound semiconductor with bandgap corresponding to just 2-5 $\mu \mathrm{m}$ mid-infrared atomspheric window is an important material of mid-infrared lasers. Research and development of new highperformance antimonide-based infrared laser are very active research subjects in recent years and researchers have made a series of important research results such as $\mathrm{AlGaAsSb} / \mathrm{InGaAsSb}$ multi-quantum well lasers [23], $\mathrm{AlSb} / \mathrm{InAs} / \mathrm{InGaSb}$ type-II quantum cascade lasers [24], "W"-shaped mid-infrared laser [25], InGaSb quantum dot lasers [26].

Antimonide-based interband cascade laser combining the advantages of quantum cascade (QC) laser and type-II quantum well interband laser has potential to achieve continuous output of high-power infrared laser at room temperature and is an international hot subject of research and development. Mid-infrared interband cascade laser made from InAs/ $\mathrm{Ga}$ (In)Sb/AlSb muti-quantum wells was reported by C. J. Hill et al of Jet Propulsion Laboratory [27]. This laser structure was grown on $\mathrm{p}-\mathrm{GaSb}$ (001) substrate by MBE as follows sequence: $0.3 \mu \mathrm{m}$ GaSb buffer layer, 2-3 $\mu \mathrm{m}$ InAs/AlSb superlattice bottom claddings, multi-quantum well $\mathrm{InAs} / \mathrm{Ga}(\mathrm{In}) \mathrm{Sb}$ /AlSb active layers ( be repeated 12-35 times), InAs/ AlSb superlattice top claddings and finally an n-type InAs cap layer. The total thickness of epitaxial layers was more than $8 \mu \mathrm{m}$. A $15 \mu \mathrm{m} \times 1.5 \mathrm{~mm}$ laser made from sample J377 lased in cw mode up to $212 \mathrm{~K}$ with an emission wavelength near $3.3 \mu \mathrm{m}$. Significant output power (over $30 \mathrm{~mW} /$ facet at $140 \mathrm{~K}$ ) has been obtained from the laser with relatively low injection currents and the laser was able to operate in pulsed mode up to $325 \mathrm{~K}$. A $15 \mu \mathrm{m} \times 1 \mathrm{~mm}$ laser made from sample J435 lased in $\mathrm{cw}$ mode at temperatures up to $165 \mathrm{~K}$ with a lasing wavelength of $5.43 \mu \mathrm{m}$ at a current of $70.5 \mathrm{~mA}$. The threshold current density increased from $43 \mathrm{~A} / \mathrm{cm}^{2}$ at $80 \mathrm{~K}$ to $470 \mathrm{~A} / \mathrm{cm}^{2}$ at $165 \mathrm{~K}$. The laser was able to operate in pulsed mode up to $325 \mathrm{~K}$ with an emission wavelength of $5.7 \mu \mathrm{m}$. However, at temperatures higher than $230 \mathrm{~K}$, the spectral linewidth is relatively broad with operation voltages higher than $10 \mathrm{~V}$.

GaInSb quantum dot surface-emitting laser (QDVCSEL) operating in optical communication wavelength band of 1.3-1.55 $\mu \mathrm{m}$ with continuous emission at room temperature by either optical pumping or current injection was reported by researchers of Japan's National Institute of Information and Communication Technology [26]. This laser mainly consists of an antimonide-based quantum dot active layer and two AlAs/GaAs superlattice distributed Bragg reflectors (DBRs). With the development of antimonide-based quantum dots, they have overcome the technical difficulty of preparing a material that emits light in the entire optical communication wavelength bands of 1.3 to $1.55 \mu \mathrm{m}$ on a GaAs substrate through conventional technologies. In particular, the obtained wavelength of $1.55 \mu \mathrm{m}$ represents the world's longest emission wavelength of existent surface-emitting laser structures based on GaAs substrate. It has great significance for mass production of low-cost surfaceemitting lasers used in next-generation ultra-high-speed optical communication technology.

High-power optically pumped semiconductor vertical external cavity surface emitting laser (VECSEL) operating at $2-\mu \mathrm{m}$ wavelength was reported by A. Härkönen $e t$ al. [28]. The device material was grown on GaSb substrate by MBE and consisted of $15 \mathrm{Ga}_{0.78} \mathrm{In}_{0.22} \mathrm{Sb}$ quantum-wells placed within a three-lambda GaSb cavity and grown on the top of an 18-pairs AlAsSb/GaSb Bragg reflector. When cooled down to $5^{\circ} \mathrm{C}$ and using 790-nm 
diode laser for optical pumping, this laser emitted up to 1 $\mathrm{W}$ of optical power in a nearly diffraction-limited Gaussian beam demonstrating the high potential of antimonide material for VECSEL fabrication. LED devices based on $\mathrm{InGaAsSb} / \mathrm{AlGaAsSb}$ multi-quantum well active region sandwiched between two AlAsSb/GaSb n- and pdoped Bragg mirrors structure has realized operation in continuous wave mode under electrical injection at room temperature and exhibited a bright emitting peak near 2.3 $\mu \mathrm{m}$ with an external quantum efficiency of $0.16 \%$ at 34 $\mathrm{A} / \mathrm{cm}^{2}$ [29]. It shows that antimonides have enormous potential in the development of new high-power, electrical injection and continuous-wave emission mid-infrared optoelectronic devices.

\subsection{Thermophotovoltaic Cells}

Thermophotovoltaic cells are similar to the solar cells that utilize the thermal infrared radiation of a heated source to directly generate electric power. The current trend of development of TPV is to develop high efficiency, low cost, narrow-bandgap ( $0.6 \mathrm{eV}$ or less) thermophotovoltaic materials and components applicable to the mid- and low-temperature radiation source $(<$ $1500^{\circ} \mathrm{C}$ ). It appears that antimonide based compounds have been one of the leading material systems for thermophotovoltaic device applications and the most studied TPV is GaSb-based InGaAsSb p-n cells fabricated by LPE, MOCVD, MBE and other methods. TPV cells based on InAsSbP, grown on InAs substrate, can have spectral responses in the 2.5-3.4 $\mu \mathrm{m}$ wavelength range and it is a hopeful research direction of having great potentials. For further details, please refer to M.G. Mauk's review paper [8].

\section{Conclusions}

As the first candidate materials for fabrication of the third generation large-scale focal plane arrays infrared (photon) detectors, integrated circuits with ultra-high speed and ultra-low power consumption and new high efficiency thermophotovoltaic devices, the research and development of antimonide based compound semiconductor materials and device applications are in the ascendant, attracting increasingly widespread concern and research interests of researchers and institutions in the world. Compared to currently more mature GaAs-based and InP-based materials growth and device manufacturing process, the growth technology of antimonide based micro-structure materials such as heterojunctions, superlattice quantum wells and self-aligned quantum dots continues to face considerable great difficulties and technical challenges and the manufacturing process of various antimonides devices are far from mature. Therefore, there are tremendous opportunities for R\&D and innovations in this area. With the gradual suppression or elimination of the adverse factors affecting device performance in narrow bandgap antimonide based compounds (such as composition segregation, Auger recombination, surface recombination, carrier absorption, etc.) by continuous optimization of material growth techniques, improving the device structure design and manufacturing processes and other technologies, we believe that in the near future, new types of high-performance antimonide devices and integrated circuits will get a wide range of important applications in the infrared imaging technologies, atmospheric environmental monitoring, biomedical diagnostics, multi-function digital radar systems, mobile communications, thermophotovoltaic power generation systems, and many other high-tech fields.

\section{Acknowledgements}

This work was supported by the National Natural Science Foundation of China (Grant No.60876004).

\section{References}

[1] B. R. Bennett, R. Magno, J. B. Boos, et al., "Antimonide-Based Compound Semiconductors for Electronic Devices: A Review," Solid-State Electronics, Vol. 49, No. 12, 2005, pp. 1875-1895.

[2] M. Razeghi, "Overview of Antimonide Based III-V Semiconductor Epitaxial Layers and Their Applications at the Center for Quantum Devices," The European Physical Journal Applied Physics, Vol. 23, No. 3, 2003, pp. 149-205.

[3] A. H. Kroemer, "The $6.1 \AA$ family (InAs, GaSb, AlSb) and its Heterostructures: a Selective Review," Physica E, Vol. 20, No. 3-4, 2004, pp. 196-203.

[4] R. M. Biefeld, "The Metal-Organic Chemical Vapor Deposition and Properties of III-V Antimony-Based Semiconductor Materials," Materials Science and Engineering $R$, Vol. 36, No. 4, 2002, pp. 105-142.

[5] C. A. Wang, "Progress and Continuing Challenges in Gasb-Based III-V Alloys and Heterostructures Grown by Organometallic Vapor-Phase Epitaxy," Journal of Crystal Growth, Vol. 272, No. 1-4, 2004, pp. 664-681.

[6] A. Rogalshi, "Material Considerations for Third Generation Infrared Photon Detectors," Infrared Physics \& Technology, Vol. 50, No. 2-3, 2007, pp. 240-252.

[7] O. Nesher and P. C. Klipstein, "High-Performance IR Detectors at SCD Present and Future," Opto-Electronics Review, Vol. 14, No. 1, 2006, pp. 61-70.

[8] M. G. Mauk and V. M. Andreev, "Gasb-Related Materials for TPV Cells," Semiconductor Science and Technology, Vol. 18, No. 5, 2003, pp. S191-S201.

[9] M. Rosker and J. Shah, "DARPA's Program on Antimonide Based Compound Semiconductors (ABCS)," IEEE GaAs Digest, 2003, p. 293.

[10] H. Toyota, T. Sasaki and Y. Jinbo, "Growth and Characterization of Gasb/Algasb Multi-Quantum Well Struc- 
tures on Si (0 0 1) Substrates," Journal of Crystal Growth, Vol. 310, No. 1, 2008, pp. 78-82.

[11] K. Zaima, R. Hasimoto, M. Ezaki, et al., "Dislocation Reduction of Gasb on Gaas by Metalorganic Chemical Vapor Deposition with Epitaxial Lateral Overgrowth," Journal of Crystal Growth, Vol. 310, No. 23, 2008, pp. 4843-4845.

[12] T. Ashley, L. Buckle, G. W. Smith, et al., "Dilute Antimonide Nitrides for Very Long Wavelength Infrared Applications," Proceedings of SPIE, Orlando, 17 May 2006, pp. 62060L.

[13] P. H. Jefferson, L. Buckle, B. R. Bennett, et al., "Growth of Dilute Nitride Alloys of Gainsb Lattice-Matched to Gasb," Journal of Crystal Growth, Vol. 304, No. 2, 2007, pp. 338-341.

[14] D. Jackrel, A. Ptak, B. Seth, et al., "Gainnassb Solar Cells Grown by Molecular Beam Epitaxy," IEEE 4th World Conference on Photovoltaic Energy Conversion. Waikoloa, HI, 2006, pp. 783-786.

[15] J. B. Hacker, J. Bergman, G. Nagy, et al., "An UltraLow Power Inas/Alsb HEMT W-Band Low-Noise Amplifier," IEEE MTT-S International Microwave Symposium, June 2005, pp. 1029-1032.

[16] M. K. Kwang, H. S. Jung, E. K. Dong, et al., "The Growth of a Low Defect Inas HEMT Structure on Si by Using an Algasb Buffer Layer Containing Insb Quantum Dots for Dislocation Termination," Nanotechnology, Vol. 20, No. 22, 2009, p. 225201.

[17] A. Rogalski. "New Material Systems for Third Generation Infrared Photo Detectors," Opto-Electron Review, Vol. 16, No. 4, 2008, pp. 458-482.

[18] M. Walther, R. Rehm, J. Schmitz, et al., "AntimonyBased Superlattices for High-Performance Infrared Imagers," Proceedings of SPIE, Orlandoof, 17 March 2008, pp. 69400A-69400A-8.

[19] E. Corbin, M. J. Shaw, M. R. Kitchin, et al., "Systematic Study of Type II Ga1-Xinxsb/Inas Superlattices for Infra-Red Detection in the 10-12 MM Wavelength Range," Semiconductor Science and Technology, Vol. 16, No. 4, 2001, pp. 263-272.
[20] C. J. Hill, J. V. Li, J. M. Mumolo, et al., "MBE Grown Type-II MWIR and LWIR Superlattice Photodiodes," Infrared Physics \& Technology, Vol. 50, No. 2-3, 2007, pp. 187-190.

[21] E. H. Aifer, J. G. Tischler, J. H. Warner, et al., "Dual Band LWIR/VLWIR Type-II Superlattice Photodiodes," Proceedings of SPIE, Orlando, 28 March 2005, Vol. 5783, pp. 112-122.

[22] M. Münzberg, R. Breiter, W. Cabanski, et al., "Inas/Gasb Type-II Short-Period Superlattices for Advanced Single And Dual-Color Focal Plane Arrays," Proceedings of SPIE, Orlando, 9 April 2007, Vol. 6542, p. 654206.

[23] Y. G. Zhang, Y. L. Zheng, C. Lin, et al., "Continuous Wave Performance and Tunability of MBE Grown 2.1 $\mu \mathrm{M}$ Ingaassb/Algaassb MQW Lasers," Chinese Physics Letters, Vol. 23, No. 8, 2006, pp. 2262-2265.

[24] A. Bauer, F. Langer, M. Dallner, et al., "Emission Wavelength Tuning of Interband Cascade Lasers in the 3-4 $\mu \mathrm{M}$ Spectral Range," Applied Physics Letters, Vol. 95, No. 25, 2009, p. 251103.

[25] W. W. Bewley, J. R. Lindle, C. S. Kim, et al., "Lifetimes and Auger Coefficients in Type-II W Interband Cascade Lasers," Applied Physics Letters, Vol. 93, No. 4, 2008, p. 041118.

[26] N. Yamamoto, "Next-Generation Optical Communications through Nanotechnology," NICT News, Vol. 353, 2005, pp. 3-4.

[27] C. J. Hill and R. Q. Yang, "MBE Growth Optimization of Sb-Based Interband Cascade Lasers," Journal of Crystal Growth, Vol. 278, No. 1-4, 2005, pp. 167-172.

[28] A. Härkönen, M. Guina, O. Okhotnikov,et al., "1-W Antimonide-Based Vertical External Cavity Surface Emitting Laser Operating At 2-MM," Optics Express, Vol.14, No. 14, 2006, pp. 6479-6484.

[29] A. Ducanchez, L. Cerutti, P. Grech, et al., "Room Temperature Continuous Wave Operation of Electrically-Injected Sb-Based RC-LED Emitting Near 2.3 MM," Superlattices and Microstructures, Vol. 44, No. 1, 2008, pp. 62-69. 